\title{
Effect of various stabilizers on the stability of lansoprazole nanosuspension prepared using high shear homogenization: Preliminary investigation
}

\author{
Shobha Ubgade*, Aditi Bapat, Vaishali Kilor \\ Department of Pharmaceutics, Gurunanak College of Pharmacy, Nagpur, India.
}

\begin{tabular}{|c|c|}
\hline ARTICLE INFO & ABSTRACT \\
\hline Received on: $28 / 02 / 2021$ & \multirow{5}{*}{$\begin{array}{l}\text { The purpose of the current research work was to prepare a nanosuspension of the model drug lansoprazole (LSP) } \\
\text { and investigate the effect of various stabilizers on the stability of the nanosuspension prepared using the high shear } \\
\text { homogenization technique. In this study, polymeric stabilizers like polyvinylpyrrolidone K-30, polyvinylpyrrolidone } \\
\text { K-90, polyvinyl alcohol, sodium alginate, and hydroxypropyl methylcellulose E15 and surfactants like sodium lauryl } \\
\text { sulfate and Tween } 80 \text { were explored. The prepared nanosuspensions were evaluated for particle size distribution } \\
\text { (PSD), polydispersity index (PI), zeta potential, and drug loading. Saturation solubility and in-vitro dissolution studies } \\
\text { of optimized nanosuspension and coarse LSP powder were also carried out to determine the extent of solubility } \\
\text { enhancement. PSD and zeta potential revealed that all the stabilizers when used alone could not significantly reduce } \\
\text { the particle size and stabilize the colloidal dispersion. However, a combination of polymeric stabilizer and surfactant } \\
\text { showed significant particle size reduction with an average particle size of } 428.5 \mathrm{~nm} \text {, PI } 0.363 \text {, and a stable zeta } \\
\text { potential value of }-25.8 \mathrm{mV} \text {. Therefore, it can be concluded that LSP nanosuspension prepared by the high shear } \\
\text { homogenization technique can be effectively stabilized by a combination of stabilizers. }\end{array}$} \\
\hline Accepted on: 04/05/2021 & \\
\hline Available online: $05 / 09 / 2021$ & \\
\hline & \\
\hline $\begin{array}{l}\text { Key words: } \\
\text { Nanosuspension, } \\
\text { lansoprazole, stability, high } \\
\text { shear homogenization, } \\
\text { stabilizer. }\end{array}$ & \\
\hline
\end{tabular}

\section{INTRODUCTION}

A nanosuspension consists of pure drug particles in the nanometer range that are usually suspended in an aqueous dispersion medium containing a surface modifier (stabilizer) to maintain the particle size in the nanometer domain (Khadka et al., 2014). The most popular techniques for producing nanosuspension are high pressure homogenization, wet media milling, antisolvent precipitation, and sonoprecipitation (Jacob et al., 2020). High shear homogenization has been used for preparing nanocrystals in combination with bottom-up techniques (Junyaprasert and Morakul, 2015) and has not been explored much as a single technique for producing drug nanocrystals. Nanonization leads to an increased interfacial tension, as well as surface area, between drug particles and the aqueous dispersion medium. As a result,

*Corresponding Author

Shobha Ubgade, Department of Pharmaceutics, Gurunanak College of Pharmacy, Nagpur, India.E-mail: shobhayadav1402@gmail.com free energy associated with the system increases, leading to a thermodynamically unstable system. In order to regain this stability, nano-sized drug particles tend to agglomerate and reduce the surface area and free energy. A surface stabilizer is required to avert this phenomenon of agglomeration and preserve the particle size in the nanometer range (Agrawal and Patel, 2011).

Lansoprazole (LSP) is a widely used proton pump inhibitor, clinically used in the treatment of diseases like gastroesophageal reflux disease, gastric and duodenal ulcers, and Zollinger-Ellison syndrome (Horn and Howden, 2005). It is a drug belonging to Biopharmaceutical Classification System (BCS) class II and is characterized by low solubility and high permeability with bioavailability of $80 \%-91 \%$ (Vora et al., 2016).

Many formulation approaches in the literature have been reported, suggesting improvement in saturation solubility and dissolution of LSP by preparing its solid dispersion (Pokharkar et al., 2011), enteric-coated microparticles for solubility enhancement and protection from acid degradation (Vora et al., 2016), enhanced dissolution, and stability of LSP by cyclodextrin inclusion complexation (Lu et al., 2012). Recently, nanotechnology- 
based techniques have also been explored for addressing both solubility and stability issues of LSP (Alai and Lin, 2014). Shende et al. (2016) reported about the formulation and characterization of engineered nanosuspension of LSP prepared by conjugating it with $\beta$-cyclodextrin and also preparing its nanosponges containing $\beta$-cyclodextrin to enhance its solubility and stability.

Although the solubility enhancement achieved in the above reports is laudable, the processed formulations are either complex in their nature or require multiple stages of processing. Therefore, there is a necessity for a simple drug delivery system which is easy to develop and improves the poor solubility of LSP. Thus, the work reported herein attempts to formulate an easy yet stable nanosuspension system. The oral solubility enhancement of LSP from stabilized nanosuspension is the prime objective with the high shear homogenizer playing pivotal role in formulation development. The current study also throws light on comparative studies of plausible effects of various stabilizers on critical parameters required for nanosuspension stabilization.

\section{MATERIALS AND METHODS}

\section{Materials}

LSP was received as a gratis sample from Alembic Pharmaceuticals Limited, Hyderabad, India. Hydroxypropyl methylcellulose E15 (HPMC E15), sodium lauryl sulfate (SLS), and Tween 80 were obtained from Research Lab Fine Chemical Industries, Mumbai. Polyvinylpyrrolidone K-30 (PVP K-30), polyvinylpyrrolidone K-90 (PVP K-90), polyvinyl alcohol (PVA), and sodium alginate were received from Himedia Laboratories Pvt. Ltd., Mumbai. All other chemicals and reagents used were of analytical grade.

\section{Selection of stabilizers}

In the present investigation, water-soluble stabilizers belonging to both classes of surfactants and polymers were selected as they form an aqueous dispersion with poorly soluble drugs much easier when compared to their hydrophobic counterparts. A total of seven stabilizers reported in the literature (Table 1) were selected to explore their effect on the stability of nanosuspension (Tuomela et al., 2016).

\section{Preparation of the nanosuspension}

Nanosuspensions of LSP were prepared by a top-down approach using the high shear homogenizer (Kinematica Polytron 3100 D). Figure 1 shows the schematic representation of the high shear homogenization process for preparation of nanosuspension. Polymeric stabilizers like PVP K-30, PVP K-90, PVA, sodium alginate, and HPMC E15 and surfactants like SLS and Tween 80 were initially used alone to prepare different batches of nanosuspensions. Furthermore, a combination of selected stabilizers at varying ratios was used for preparing the nanosuspension. The selection of stabilizers for use in combination was based on the results of mean particle size and zeta potential yielded by stabilizers when used alone. Accurately weighed amounts of stabilizers (polymer/surfactant) were added and dissolved in distilled water to prepare different stabilizer solutions. An alkaline stabilizer sodium bicarbonate at a concentration of $8.4 \% \mathrm{w} / \mathrm{v}$ was added in all the formulations (Morrison et al., 2013). The drug was dispersed in a cold stabilizer solution and homogenized using the high shear homogenizer to obtain nanosuspension. The resulting nanosuspensions were stored in amber-colored glass containers at $4^{\circ} \mathrm{C}$ till further evaluation (Tables 2 and 3) (Fig. 2).

\section{Characterization of LSP nanosuspension}

\section{Particle size distribution (PSD) and polydispersity index (PI)}

The particle size analysis was carried out by photon correlation spectroscopy (PCS) using the Horiba nanoparticle analyzer (Nanopartica SZ-100). This analysis reports the mean particle size (z-average), PI. Particle size is determined

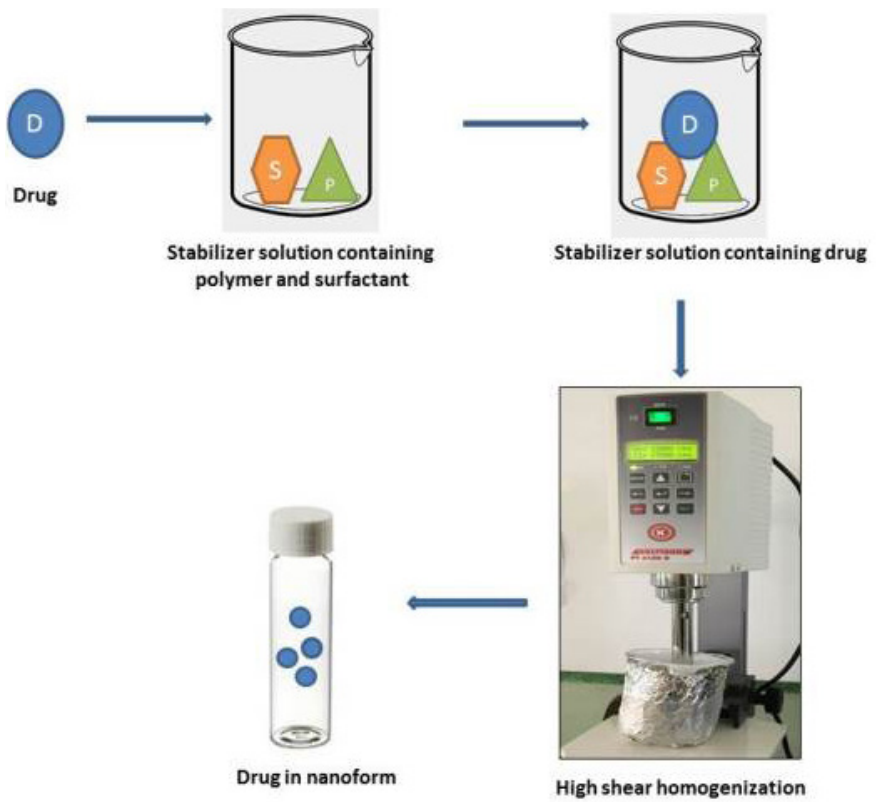

Figure 1. Schematic representation of production of nanosuspension using high shear homogenization.

Table 1. Screened stabilizers for preparing nanosuspensions.

\begin{tabular}{llcc}
\hline Name & Category & Molecular weight $\mathbf{( g / m o l )}$ & Stabilization mechanism \\
\hline sodium dodecyl sulfate (SDS)/SLS & Anionic surfactant & 288 & Electrostatic \\
Tween 80 & Non-ionic surfactant & 1,310 & Steric \\
HPMC E15 & Semisynthetic non-ionic polymer & $1,261.4$ & Steric \\
PVP K-30 & Synthetic linear polymer & 40,000 & Steric \\
PVP K-90 & Synthetic linear polymer & 360,000 & Steric \\
PVA & Synthetic linear polymer & 80,000 & Steric \\
Sodium alginate & Semisynthetic ionic polymer & 216.12 & Electrosteric \\
\hline
\end{tabular}


Table 2. Composition of various batches of LSP nanosuspension.

\begin{tabular}{cccccc}
\hline $\begin{array}{c}\text { Formulation } \\
\text { Code }\end{array}$ & $\begin{array}{c}\text { Drug(\% } \\
\boldsymbol{w} / \boldsymbol{v})\end{array}$ & $\begin{array}{c}\text { Type of } \\
\text { stabilizer }\end{array}$ & $\begin{array}{c}\text { Drug: stabilizer } \\
\text { ratio }\end{array}$ & $\begin{array}{c}\text { Homogenization } \\
\text { speed (RPM) }\end{array}$ & $\begin{array}{c}\text { Homogenization } \\
\text { Time (hour) }\end{array}$ \\
\hline NSP1 & 1 & HPMC E15 & $1: 1$ & 10,000 & 1 hour \\
NSP2 & 1 & PVP K-30 & $1: 1$ & 10,000 & 1 hour \\
NSP3 & 1 & PVP K-90 & $1: 1$ & 10,000 & 1 hour \\
NSP4 & 1 & Sodium alginate & $1: 1$ & 10,000 & 1 hour \\
NSP5 & 1 & PVA & $1: 1$ & 10,000 & 1 hour \\
NSS1 & 1 & SLS & $1: 1$ & 10,000 & 1 hour \\
NSS2 & 1 & Tween 80 & $1: 1$ & 10,000 & 1 hour \\
\hline
\end{tabular}

Table 3. Composition of LSP nanosuspension containing selected stabilizers in combination.

\begin{tabular}{ccccccc}
\hline $\begin{array}{c}\text { Formulation } \\
\text { Code }\end{array}$ & $\begin{array}{c}\text { Drug } \\
(\boldsymbol{\%} \boldsymbol{w} / \boldsymbol{v})\end{array}$ & Type of stabilizer & $\begin{array}{c}\text { Amount of } \\
\text { stabilizer }(\boldsymbol{\%} \boldsymbol{w} / \boldsymbol{v})\end{array}$ & $\begin{array}{c}\text { Stabilizer } \\
\text { ratio }\end{array}$ & RPM & Time \\
\hline NSC1 & 1 & HPMC E15 + SLS & $1(0.5+0.5)$ & $1: 1$ & 10,000 & 1 hour \\
NSC2 & 1 & HPMC E15+ SLS & $1.5(0.5+1)$ & $1: 2$ & 10,000 & 1 hour \\
NSC3 & 1 & HPMC E15 + SLS & $1(0.5+0.5)$ & $1: 1$ & 15,000 & 1 hour \\
NSC4 & 1 & HPMC E15+ SLS & $1.5(0.5+1)$ & $1: 2$ & 15,000 & 1 hour \\
\hline
\end{tabular}

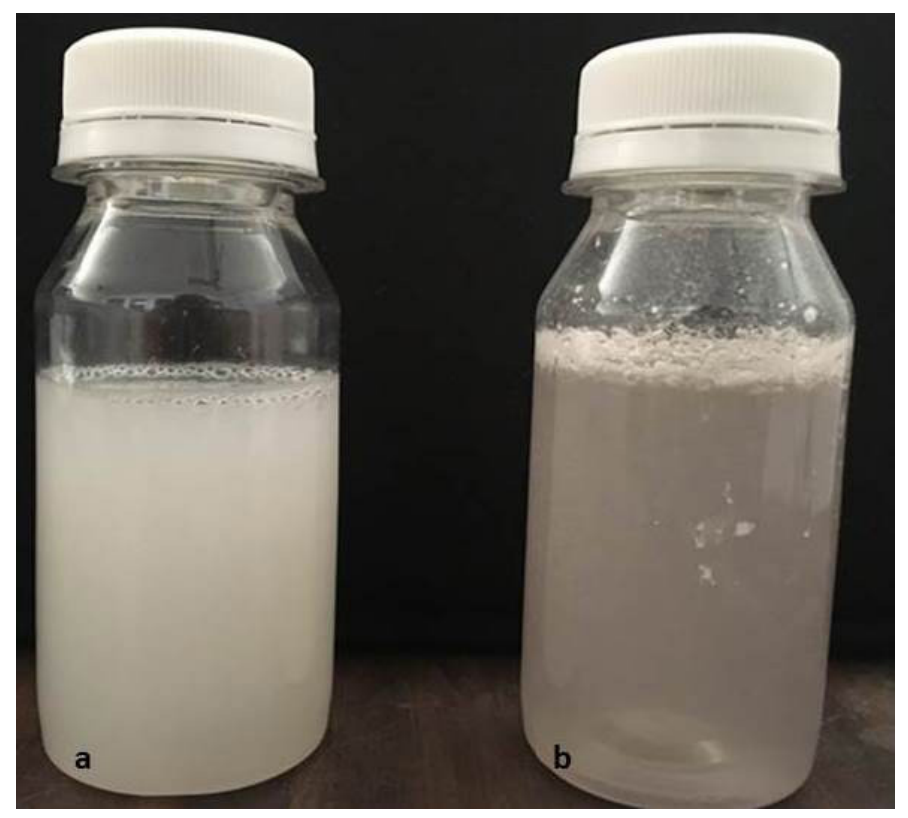

Figure 2. (a) Optimized nanosuspension and (b) Pure drug suspension.

by measuring the changes in the intensity of light scattered from the sample of nanosuspension. This technique is also commonly known as dynamic light scattering and quasi-elastic light scattering. In this study, samples of nanosuspensions were diluted to an appropriate concentration using distilled water as the dispersant and were measured at $25^{\circ} \mathrm{C}$ and at a scattering angle of $90^{\circ}$. All measurements were carried out in triplicate and the mean values and standard deviations are reported.

\section{Zeta potential}

The charge on the surface of particles is characterized by calculating the zeta potential and is an indicator of dispersion stability. Large magnitude values of zeta potential indicate higher stability of a suspension. For the purpose of determining the stability of prepared nanosuspension imparted by various stabilizers, the zeta potential of all the samples was measured. Zeta potential was also determined by PCS using Horiba nanoparticle analyzer (Nanopartica SZ-100) at $25^{\circ} \mathrm{C}$. In this process, the sample to be analyzed is placed in a disposable cell and the zeta potential is calculated based on the extent of particle electrophoretic mobility. All the zeta potential measurements of nanosuspensions containing different stabilizers were carried out in triplicate with the mean values and standard deviations reported.

\section{Percent drug loading}

Drug loading in all the nanosuspension formulations was determined spectrophotometrically. An aliquot $(1 \mathrm{ml})$ of nanosuspension was diluted up to $10 \mathrm{ml}$ with methanol and filtered through $0.45 \mu \mathrm{m}$ filter paper to assure the clarity of the sample for assay. The sample was analyzed using UV-visible spectrophotometer (Shimadzu-1700, Japan) at $\lambda \max$ of 284 nm using methanol as a blank. Each sample was prepared and analyzed in triplicate. The amount of LSP contained in each formulation was determined using a standard curve prepared from a known standard solution. All the drug content measurements of nanosuspensions containing different stabilizers were made in triplicate with the mean values and standard deviations reported.

\section{Saturation solubility determination}

Saturation solubility was assessed for both unprocessed pure drug and optimized nanosuspension. Accurately weighed 10 $\mathrm{mg}$ of pure drug and nanosuspension equivalent to $10 \mathrm{mg}$ of LSP was separately introduced into a $25 \mathrm{ml}$ stoppered conical flask containing $10 \mathrm{ml}$ of distilled water. The sealed flask was placed in a rotary shaker at $37^{\circ} \mathrm{C}$ and equilibrated for 6 hours. The contents were then filtered, and the suitably diluted samples were analyzed using UV-visible spectrophotometer (Shimadzu-1700, Japan) at $285 \mathrm{~nm}$, against distilled water as a blank. Each sample was prepared and analyzed in triplicate. 


\section{Dissolution studies}

In-vitro dissolution studies were carried out for coarse LSP powder and its optimized nanosuspension using United States Pharmacopoeia apparatus 2 (paddle method) in triplicate. Paddle rotation speed of $50 \mathrm{rpm}$ and a temperature of $37^{\circ} \mathrm{C} \pm 0.5^{\circ} \mathrm{C}$ were used in each study. Coarse LSP powder and nanosuspension (equivalent to $30 \mathrm{mg}$ of LSP) were dispersed in $900 \mathrm{ml}$ of dissolution medium ( $\mathrm{pH} 6.8$ buffer solution). At predetermined time intervals, $5 \mathrm{ml}$ of sample was withdrawn and the dissolution medium was kept constant by refilling it with fresh buffer solution to achieve sink conditions. The collected samples were filtered, suitably diluted, and analyzed at $285 \mathrm{~nm}$ using UV-visible spectrophotometer.

\section{Short-term physical stability}

Physical stability of the optimized nanosuspension was evaluated for a period of up to 1 month at refrigerated conditions $\left(2-8^{\circ} \mathrm{C}\right)$. Nanosuspension was stored in a closed amber-colored glass vial. The stability was assessed in terms of PSD, zeta potential, and percent drug loading.

\section{RESULT AND DISCUSSION}

\section{Wet milling using high shear homogenization for preparation of nanosuspension}

A simple, easy, and universal milling technique to prepare nanosuspension is required for pharmaceutical development (Niwa et al., 2011). The high shear homogenization process is a simple form of wet milling where milling of a solid suspended in liquid is carried out. This process utilizes high shear devices having a high-speed mixing element in the form of a rotor and a static element as the stator, where the former is in close proximity to the latter. The shear generated by these devices is of a much higher magnitude when compared to the conventional stirring devices. The rotor and stator actions result in the creation of shear stress, turbulence, and cavitation forces which are responsible for high shear mixing and size reduction (Malamatari et al., 2018). This device operates at varied homogenization speed (up to 30,000 rpm) to yield and control the particle size in suitable range for producing nanosuspension.

\section{Stabilization mechanisms}

Regardless of the formulation mechanism, nano-sized drug crystals formed in the aqueous dispersion tend to come together and agglomerate. This phenomenon is hindered by virtue of conferred protection rendered in the presence of surface-active agents which reduces the free energy of the system and stabilizes it (Rabinow, 2004). Owing to their complementary properties, stabilizers of two classes were utilized in this study: surfactants which effect an electrostatic repulsion and polymers which effect a steric repulsion. Both electrostatic and steric stabilization mechanisms are warranted by combining polymers and ionic surfactants. Besides stabilizer selection, several other factors, like molecular weight, functional groups, morphology of polymers, and ratio of hydrophilic:hydrophobic part, in a stabilizer molecule also affects the particle size and stability of nanocrystals (Tuomela et al., 2016). Various stabilizers explored in this study showed a huge variance with respect to key characterization parameters of nanosuspension. It was observed that stabilizers having high molecular weight (PVP K-30, PVP K-90, and PVA) were less effective in reducing particle size below $1,000 \mathrm{~nm}$ when compared to low molecular weight stabilizers (SLS, Tween 80, and HPMC E15), except sodium alginate (molecular weight $216.12 \mathrm{~g} / \mathrm{mol}$ ). Stabilization of the colloidal system is not only important for yielding nano-sized drug crystals and thermodynamically stable systems, but also for storage and dissolution of nano-crystal-based solid dosage forms (Shete et al., 2014).

successful production of stable nanosuspension using a combination of stabilizers have been reported in the literature earlier for poorly water soluble drug candidates. The use of an anionic surfactant (SDS) along with a polymeric stabilizer (HPMC) resulted in better nano-sizing capability when compared to an individual stabilizer (Lestari et al., 2015). A multiple stabilizer system was expected to gain the combined advantages of both steric and electrostatic stabilization in achieving a more efficient particle size of Itraconazole during homogenization (Sun et al., 2011). Celecoxib nanosuspension was prepared using a combination of SDS and PVP, the rationale was based on a combined electrostatic and steric stabilization (Dolenc et al., 2009). Stable nanosuspensions of BCS class II drugs, naproxen, fenofibrate, and griseofulvin were prepared by wet stirred media milling using HPMC and SDS as stabilizers (Figueroa et al., 2012). Cellulosic polymer hydroxy propyl cellulose and anionic surfactant SDS exhibited promising synergistic effects by giving finer milled particle sizes for five drugs (Bilgili et al., 2016). Hence, various formulators have pointed out the potential synergistic stabilization effects derived from the use of a combination of stabilizers.

\section{Preparation of LSP nanosuspension}

Nanosuspension was prepared initially by using single stabilizers in the dispersion at a concentration of $1 \%$ $w / v$. Furthermore, based on the preliminary results, polymeric stabilizer HPMC E15 and anionic surfactant SLS were selected as the final stabilizers and their synergistic effect on the stability of nanosuspension was determined. The amount of stabilizers, which was required to support the size reduction and stabilize nanodispersion, was investigated to be in the minimum range to avoid toxicity attributed to their surface-active properties (Niwa et al., 2011). About $1 \% w / v$ of LSP was added to the nanosuspension. Various compositions of both HPMC E15 and SLS (as shown in Table 3) were used to prepare batches of nanosuspension at a homogenization speed of 10,000 and 15,000 rpm for 1 hour, respectively. LSP is a heat- and light-sensitive drug molecule; therefore, the operating conditions included a short duration of processing time up to 1 hour and maintenance of cold conditions around the homogenization assembly with minimal exposure to light.

Nanosuspension of LSP was successfully prepared with the high shear homogenization technique using the combination of a polymeric stabilizer HPMC E15 and an anionic surfactant SLS. Homogenization speed was found to be important in case of production of nanosuspension as increased speed from 10,000 to $15,000 \mathrm{rpm}$ showed a decrease in particle size.

Nanosuspension is colloidal dispersion having a particle size range between 1 and 1,000 nm (Rabinow, 2004), wherein nano-sized drug particles are suspended in an aqueous solution of the stabilizer. Sedimentation is a common phenomenon that occurs in case of dispersions. Batches of polymeric stabilizers were found 
to be turbid and flocculated while that of surfactants were a bit hazy but not flocculated. Since the drug is insoluble in water, it may not produce clear visibility like molecular dispersion. Large-sized particles may cause agglomeration and flocculation as observed in most of the polymeric stabilizers under study. However, a little hazy and turbid appearance may be a result of suspended smallsized particles in the dispersion. With regards to the combination of the polymer and surfactant also, clear nanosuspensions were not obtained either.

\section{PSD and PI}

PSD is an important and integral evaluation parameter for nanosuspension. Since reduced particle size in a submicron range helps in improving solubility of pure drug, thereby increasing the dissolution and bioavailability, it becomes an essential quality attribute of nanodispersion. Moreover, the distribution of particle size in the formulation is also assessed as PI which gives a degree of PSD. The PI is an important evaluation parameter that controls the physical stability of nanosuspensions and should be ideally on a lower side for achieving long-term stability of a nanosuspension. A PI value of $0.1-0.25$ indicates a narrow size distribution and a PI value above 0.5 indicates a broad distribution (Patravale et al., 2004). A narrow size distribution is recommended to prevent particle growth due to Ostwald ripening and also maintaining the stability of nanosuspensions. Particle size data and PI results for nanosuspension are tabulated in Table 4.

The mean particle size (z-average) of different batches of LSP nanosuspensions prepared using various stabilizers was found to be in the range of 428-6,608 $\mathrm{nm}$. The results of PSD of LN indicate that polymeric stabilizers, except HPMC E15, were less effective (z-average $>5,000 \mathrm{~nm}$, PI $>1$ ) than surfactants (z-average $<2,000 \mathrm{~nm}$, PI $<1.5$ ). As evident from the results, not all the stabilizers were able to reduce the particle size of LSP successfully. Sodium alginate, PVP K-90, PVP K-30, and PVA could not reduce the particle size in the submicron range. On the other hand, nanosuspension prepared using single stabilizers like HPMC E15, SLS, and Tween 80 could significantly reduce the particle size to nanorange.

However, when a combination of HPMC E15 and SLS was used as stabilizer, the particle size was reduced drastically when compared to individual results of the stabilizers. As compared to polymers, surfactants can provide efficacious wetting of the drug particles (Sun et al., 2011). This would lead to the formation of a better dispersion of the drug particles and a significant particle size reduction (Fig. 3). The amount of surfactant played a significant role in the size reduction process as evident from the results. SLS is an ionic surfactant and in this study it is used as a stabilizer for charged stabilization of the homogenized and suspended nanoparticles. The homogeneity in particle size is important to avoid agglomeration and the protective layer of polymeric stabilizers in the form of stearic barrier hinders the process of Ostwald ripening and subsequent agglomeration. PI values of nanosuspensions prepared using combination of polymer and surfactant were found to be in the range of $0.363-1$.

\section{Zeta potential}

Determination of zeta potential is essential as it indicates the physical stability of the colloidal dispersion. Production of

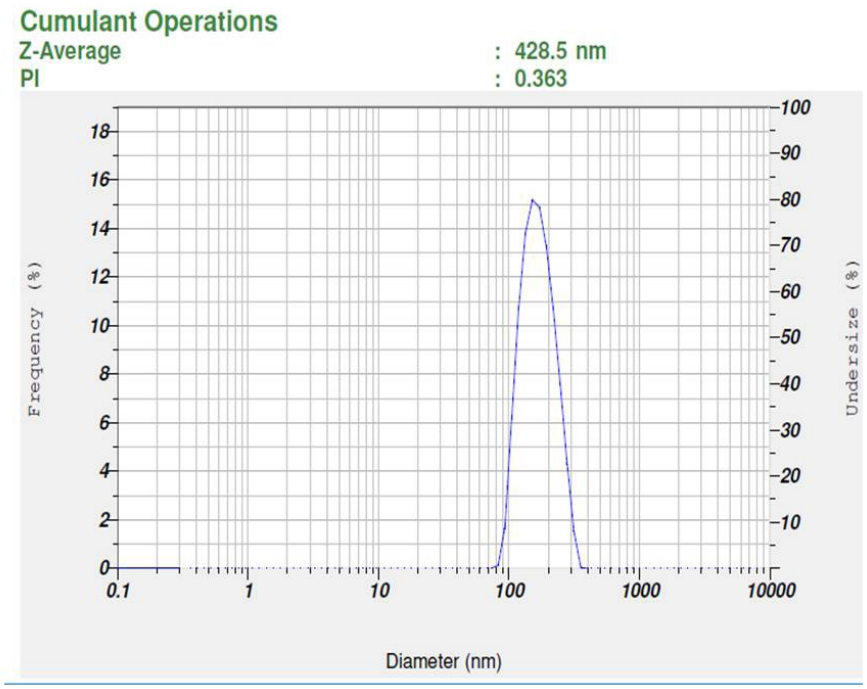

Figure 3. Particle size distribution of optimized batch.

Table 4. Results of characterization parameters of LSP nanosuspension.

\begin{tabular}{ccccc}
\hline Formulation code & Mean particle size $(\mathbf{n m})$ & PI & Zeta potential $(\mathbf{m V})$ & \% Drug loading \\
\hline NSP1 & $1,002 \pm 3.5$ & $0.659 \pm 0.09$ & $-11.1 \pm 0.7$ & $53 \pm 1.14$ \\
NSP2 & $5,248 \pm 23.8$ & $1.0 \pm 0.07$ & $-2.7 \pm 0.2$ & $28 \pm 2.31$ \\
NSP3 & $6,608 \pm 34.5$ & $5.5 \pm 0.9$ & $-5.6 \pm 0.5$ & $21 \pm 1.08$ \\
NSP4 & $6,393 \pm 17.6$ & $3.1 \pm 1.2$ & $-1.1 \pm 0.3$ & $25 \pm 1.65$ \\
NSP5 & $\ldots \ldots .^{\mathrm{a}}$ & $\ldots \ldots \ldots{ }^{\mathrm{a}}$ & $\ldots \ldots \ldots{ }^{\mathrm{a}}$ & $15 \pm 2.13$ \\
NSS1 & $859.6 \pm 9$ & $0.612 \pm 0.03$ & $-21.3 \pm 2$ & $61 \pm 1.43$ \\
NSS2 & $1,692.9 \pm 11.7$ & $1.070 \pm 0.5$ & $-15.4 \pm 1.5$ & $57 \pm 1.83$ \\
NSC1 & $512.9 \pm 4.6$ & $0.417 \pm 0.02$ & $\ldots \ldots \ldots \ldots$ & $70 \pm 2.11$ \\
NSC2 & $642.9 \pm 8.5$ & $1.044 \pm 0.04$ & $\ldots \ldots \ldots \ldots$ & $69 \pm 1.98$ \\
NSC3 & $428.5 \pm 5.5$ & $0.363 \pm 0.06$ & $-25.8 \pm 1.8$ & $81 \pm 1.02$ \\
NSC4 & $608.9 \pm 16.2$ & $1.08 \pm 0.7$ & $\ldots \ldots \ldots \ldots$ & $67 \pm 3.01$ \\
\hline
\end{tabular}

Mean $\pm \mathrm{SD}(n=3)$.

${ }^{a}$ Not detected in nanorange. 
nano-sized particles creates high-energy surfaces, if appropriate stabilization does not take place. This eventually leads to the formation of aggregates, Ostwald ripening effect, and an unstable colloidal dispersion. The importance of stabilization increases with the decrease in particle size and increase in free surface energy (Wang et al., 2013). Stability is essential for both the formation of nanosuspensions and storage stability (Verma et al., 2011). A drug nanocrystal is surrounded by a protective layer of stabilizers present in the dispersion. Stabilizers can be divided primarily into two categories: polymers and surfactants. These can be ionic or non-ionic in nature and impart stability either by steric hindrance or electrostatic forces. The nano-sized particles possess high surface energy and are prone to agglomeration or aggregation of drug crystals. Stabilizers prevent agglomeration or aggregation to yield a physically stable formulation by providing steric or ionic barriers (Tuomela et al., 2016). The zeta potential of a nanosuspension depends on the stabilizer and the drug itself. A minimum zeta potential of $\pm 30 \mathrm{mV}$ is required for an electrostatically stabilized nanosuspension, whereas a minimum zeta potential of $\pm 20 \mathrm{mV}$ is desirable in the case of a combined electrostatic and steric stabilization (Patravale et al., 2004). Zeta potential values (as shown in Table 4) of different batches of nanosuspension indicate poor physical stability. Both polymeric stabilizers and surfactants incorporated alone could not completely stabilize the nanosuspension of LSP. However, the combination of polymer and surfactant could stabilize the system very well as shown in the table with zeta potential of $-25.8 \mathrm{mV}$ (NSC3) (Fig. 4). The adsorption of polymers onto the surface of nano-sized drug particles provides steric hindrance and forms a mechanical barrier to avoid particle aggregation. Surfactants have good wetting properties and are relatively small compared to the large polymeric chains; therefore, they can quickly occupy the newly built drug surfaces produced in the nano-milling process. This type of effect initially provides substantial protection against particle agglomeration. However, steric hindrance offered by a polymer gives rise to better and long-term stability. Therefore, many nanosuspensions manufactured by wet ball milling are based on electrosteric stabilization. This is attained by combining a surfactant with a polymer (Bilgili et al., 2016).

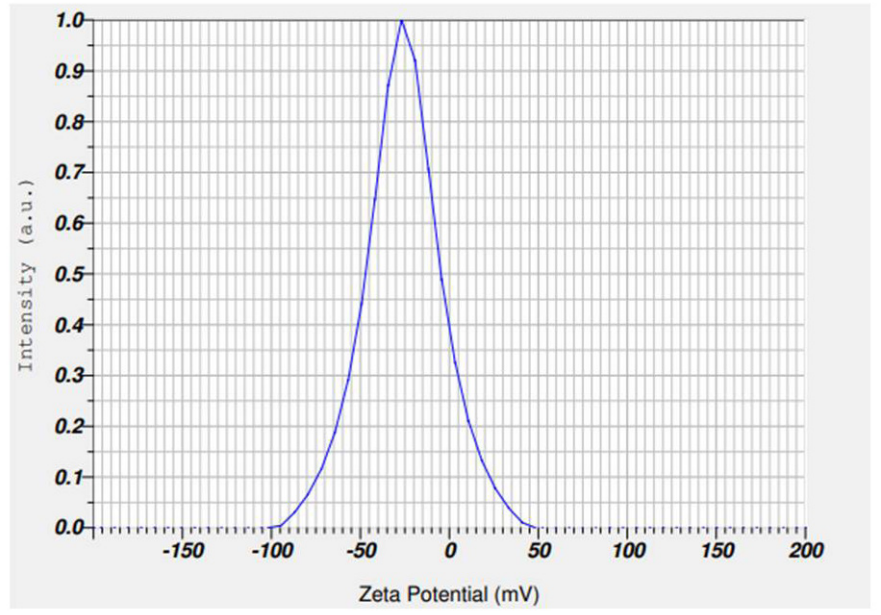

Figure 4. Zeta potential of optimized batch.
Optimized formulation NSC3 contains both polymeric stabilizer and surfactant which is responsible for keeping particles well dispersed via electrostearic stabilization and rendering more stable nanosuspension of LSP.

\section{Drug loading}

The amount of LSP present as nano-sized drug particle in the dispersion was measured by UV-visible spectroscopy. Drug loading of LSP in nanosuspension was found to be in the range of $15 \%-81 \%$. Optimized nanosuspension formulation NSC3 showed around $81 \%$ of LSP in suspended form. Larger particles that were not in the submicron range must have settled down at the bottom, leading to low drug loading in various formulations of the nanosuspension.

\section{Saturation solubility determination}

The results of saturation solubility of plain drug (LSP) and LSP nanosuspension revealed a saturation solubility of $30.15 \mu \mathrm{g} / \mathrm{ml}(0.03 \mathrm{mg} / \mathrm{ml})$ and $196.64 \mu \mathrm{g} / \mathrm{ml}(0.196 \mathrm{mg} / \mathrm{ml})$, respectively (Table 5). Thus, the saturation solubility of LSP as a nanosuspension is 6.52-folds higher than that of plain LSP. In the present study, the particle size of the drug has been reduced and hence an increased saturation solubility has been observed.

\section{Dissolution studies}

Raw LSP showed a very low dissolution rate; only $12 \%$ of the drug was dissolved in the first 10 minutes. On the other hand, the LSP-optimized nanosuspension prepared using a combination of stabilizers showed around $80 \%$ drug release in the first 10 minutes. Rapid dissolution shown by the nanosuspension formulation indicates the presence of increased surface area of the

Table 5. Saturation solubility data of LSP nanosuspension and pure drug.

\begin{tabular}{ccc}
\hline Formulation code & Formulation type & Saturation solubility(mg/ml) \\
\hline NSC3 & Nanosuspension & $0.196 \pm 0.46$ \\
- & Pure drug & $0.03 \pm 0.19$ \\
\hline
\end{tabular}

Mean $\pm \operatorname{SD}(n=3)$.

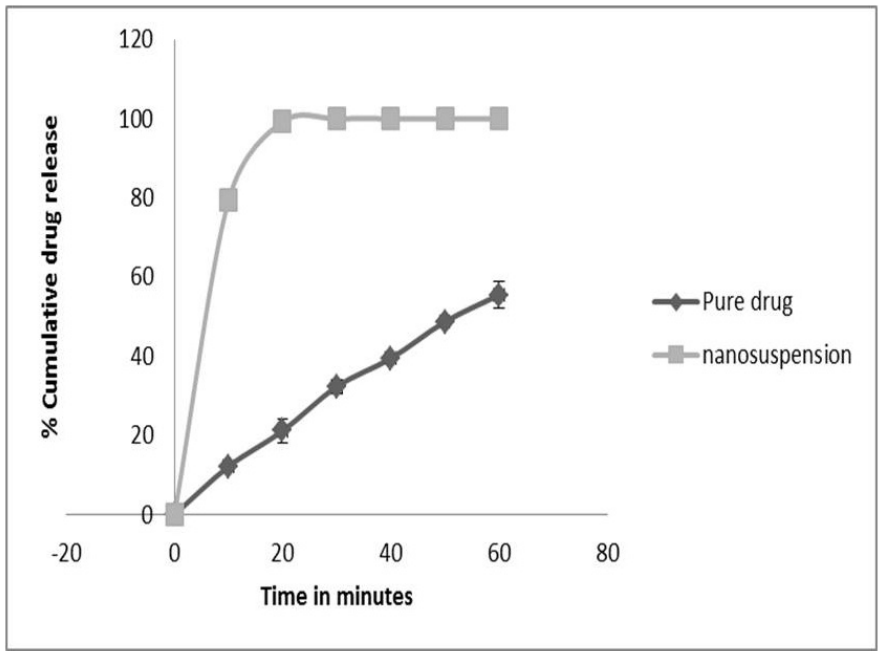

Figure 5. In vitro release profiles of pure drug and nanosuspension. 
Table 6. Storage stability data of optimized LSP nanosuspension (NSC3).

\begin{tabular}{ccccc}
\hline Time Interval & Mean particle size (nm) & PI & Zeta potential $(\mathbf{m V})$ & \% Drug loading \\
\hline At 0 month & $428.5 \pm 5.5$ & $0.363 \pm 0.06$ & $-25.8 \pm 1.8$ & $81 \pm 1.02$ \\
At 1 month & $435.8 \pm 7.5$ & $0.371 \pm 0.03$ & $-25.3 \pm 2.1$ & $80 \pm 2.1$ \\
\hline
\end{tabular}

Mean $\pm \operatorname{SD}(n=3)$.

drug particles in the nano-sized range and the enhanced saturation solubility accelerated the dissolution rate. The results of the invitro dissolution studies are shown in Figure 5.

\section{Short-term physical stability}

The optimized nanosuspension formulation showed good physical stability. No considerable change in particle size, zeta potential, and percent drug loading was observed up to one month. when it was stored in refrigerated conditions (Table 6). Physical stability of nanosuspensions is due to the absence of Ostwald ripening phenomenon. Ostwald ripening can be circumvented by two ways. Firstly, the drug is poorly soluble in the stabilizer solution and thus leads to very little changes in the dissolved concentration during the production process. Secondly, the particles are homogenous in size (Möschwitzer et al., 2004).

\section{CONCLUSION}

Nanosuspension of LSP was successfully prepared using high shear homogenization as a wet milling technique. Preliminary investigative studies and evaluation of critical parameters like zeta potential, PSD, and PI indicate that the combination of HPMC E15 and SLS exhibits a narrow range of size distribution of nanocrystals with a mean particle size of $428.5 \mathrm{~nm}$ and a stable zeta potential value $-25.8 \mathrm{mV}$. Moreover, for the same batch, stability studies at an ambient temperature increased the propensity of maintenance of drug content and particle size retention without agglomeration. This has been confirmed by electrosteric stabilization of the nanosuspension due to higher electrostatic repulsive forces between the particles, as well as enhanced steric hindrance from the adsorbed polymer. As the effect of stabilizer type and ratio used provided an important direction for optimization of formulation parameters, equivalent concentrations of HPMC E15 and SLS have reinforced their synergistic action with respect to the mean particle size, PI, drug loading, and zeta potential as evident from the results. Furthermore, the findings from envisaged research suggest high shear homogenization as a reasonable, novel, and alternative top-down approach for the production of stable nanosuspension of LSP. However, further studies with suitable tools, like design of experiment, are imperative in order to explore the formulation and process variables minutely.

\section{ACKNOWLEDGMENTS}

We would like to acknowledge Dr. A. M. Ittadwar, Principal, Gurunanak College of Pharmacy, Nagpur, for providing infrastructural facilities to carry out the research work. We are also thankful to Dr. Vipin Dhote, Principal VNS Institute of Pharmacy, Bhopal, India, for providing facilities to carry out particle size analysis using Horiba Nanosizer, and Alembic Pharmaceuticals Limited, Hyderabad, India, for providing gift sample of API LSP. We would like to appreciate the efforts of Mr. Keshav Moharir and Dr. Sumit Arora for their valuable guidance in preparation and editing of the manuscript.

\section{CONFLICT OF INTEREST}

The authors report no conflicts of interest. The authors alone are responsible for the content and writing of this article.

\section{FUNDING}

None.

\section{AUTHOR CONTRIBUTIONS}

All authors made substantial contributions to conception and design, acquisition of data, or analysis and interpretation of data; took part in drafting the article or revising it critically for important intellectual content; agreed to submit to the current journal; gave final approval of the version to be published; and agree to be accountable for all aspects of the work. All the authors are eligible to be an author as per the international committee of medical journal editors (ICMJE) requirements/guidelines.

\section{ETHICAL APPROVAL}

Not applicable.

\section{REFERENCES}

Agrawal Y, Patel V. Nanosuspension: an approach to enhance solubility of drugs. J Adv Pharm Technol Res, 2011; 2(2):81.

Alai M, Lin WJ. Novel lansoprazole-loaded nanoparticles for the treatment of gastric acid secretion-related ulcers: in vitro and in vivo pharmacokinetic pharmacodynamic evaluation. AAPS J, 2014; 16(3): 361-72.

Bilgili E, Li M, Afolabi A. Is the combination of cellulosic polymers and anionic surfactants a good strategy for ensuring physical stability of BCS Class II drug nanosuspensions? Pharm Dev Technol, 2016; 21(4):499-510.

Dolenc A, Kristl J, Baumgartner S, Planinsek O. Advantages of celecoxib nanosuspension formulation and transformation into tablets. Int $\mathrm{J}$ Pharm, 2009; 376(1-2):204-12.

Figueroa LS, Bhakay A, Jackeline I, Rozo J, Pandya N, Romanach RJ, Kohn BM, Iqbal Z, Bilgili E, Dave RN. Preparation and characterization of hydroxypropyl methyl cellulose films containing stable BCS Class II drug nanoparticles for pharmaceutical applications. Int J Pharm, 2012; 423(2):496-508.

Horn JR, Howden CW. Similarities and differences among delayed-release proton-pump inhibitor formulations. Aliment Pharmacol Ther Suppl, 2005; 22(3):20-4.

Jacob S, Nair AB, Shah J. Emerging role of nanosuspensions in drug delivery systems. Biomater Res, 2020; 24(1):1-16.

Junyaprasert VB, Morakul B. Nanocrystals for enhancement of oral bioavailability of poorly water-soluble drugs. Asian J Pharm Sci, 2015; 10(1):13-23.

Khadka P, Ro J, Kim H, Kim I, Kim JT, Kim H, Cho MJ, Yun G, Lee J. Pharmaceutical particle technologies: an approach to improve drug solubility, dissolution and bioavailability. Asian J Pharm Sci, 2014; 9(6):304-16.

Lestari MLAD, Müller RH, Möschwitzer JP. Systematic screening of different surface modifiers for the production of physically stable nanosuspensions. J Pharm Sci, 2015; 104(3):1128-40. 
Lu Y, Guo T, Qi J, Zhang J, Wu W. Enhanced dissolution and stability of lansoprazole by cyclodextrin inclusion complexation: preparation, characterization, and molecular modeling. AAPS PharmSciTech, 2012; 13(4):1222-9.

Malamatari M, Taylor KMG, Malamataris S, Douroumis D, Kachrimanis K. Pharmaceutical nanocrystals: production by wet milling and applications. Drug Discov Today, 2018; 23(3):534-47.

Morrison JT, Lugo RA, Thigpen JC, Brown SD. Stability of extemporaneously prepared Lansoprazole suspension at two temperatures. J Pediatr Pharmacol Ther, 2013; 18(2):122-7.

Möschwitzer J, Achleitner G, Pomper H, Müller RH. Development of an intravenously injectable chemically stable aqueous omeprazole formulation using nanosuspension technology. Eur J Pharm Biopharm, 2004; 58(3):615-9.

Niwa T, Miura S, Danjo K. Universal wet-milling technique to prepare oral nanosuspension focused on discovery and preclinical animal studies - development of particle design method. Int J Pharm, 2011; 405(12):218-27.

Patravale VB, Date AA, Kulkarni RM. Nanosuspensions: a promising drug delivery strategy. J Pharm Pharmacol, 2004; 56(7):827-40.

Pokharkar V, Mendiratta C, Kadam V. Lansoprazole solid dispersion using a novel amphiphillic polymer soluplus ${ }^{\circledR}$. J Chem Pharm Res, 2011; 3(6):536-43.

Rabinow BE. Nanosuspensions in drug delivery. Nat Rev Drug Discov, 2004; 3(9): 785-96.

Shende P, Chaphalkar R, Deshmukh K, Gaud RS. Physicochemical investigation of engineered nanosuspensions containing model drug, Lansoprazole. J Dispers Sci Technol, 2016; 37(4):504-11.
Shete G, Jain H, Punj D, Prajapat H, Akotiya P, Bansal AK. Stabilizers used in nano-crystal based drug delivery systems. J Excip Food Chem, 2014; 5(4):184-209.

Sun W, Mao S, Shi Y, Li LC, Fang L. Nanonization of itraconazole by high pressure homogenization: stabilizer optimization and effect of particle size on oral absorption. J Pharm Sci, 2011; 100(8):3365-73.

Tuomela A, Hirvonen J, Peltonen L. Stabilizing agents for drug nanocrystals: effect on bioavailability. Pharmaceutics, 2016; 8(2):16.

Verma S, Kumar S, Gokhale R, Burgess DJ. Physical stability of nanosuspensions: investigation of the role of stabilizers on Ostwald ripening. Int J Pharm, 2011; 406(1-2):145-52.

Vora C, Patadia R, Mittal K, Mashru R. Formulation development, process optimization, and in vitro characterization of spraydried lansoprazole enteric microparticles. Sci Pharm, 2016; 84(2):393-408.

Wang Y, Zheng Y, Zhang L, Wang Q, Zhang D. Stability of nanosuspensions in drug delivery. J Control Release, 2013; 172(3):1126-41.

\section{How to cite this article:}

Ubgade S, Bapat A, Kilor V. Effect of various stabilizers on the stability of lansoprazole nanosuspension prepared using high shear homogenization: Preliminary investigation. J Appl Pharm Sci, 2021; 11(09):085-092. 